\title{
Determination of fracture toughness using the compression fracture technique
}

\author{
Carl M. Cady ${ }^{1 *}$ and Cheng Liu ${ }^{1}$ \\ ${ }^{1}$ MST-8, MS-G755, Los Alamos National Laboratory, Los Alamos NM 87545, U.S.A.
}

\begin{abstract}
Many engineering materials of importance can be mechanically characterized as brittle or quasi-brittle solids. Examples include many hard polymers, ceramic composites, and low ductility metals. Fracture toughness is the measure of crack extension as a function of applied load and the resistance of the deforming material to the advance of cracking. In this investigation we use digital image correlation (DIC) for observing and studying the process of macroscopic crack initiation and propagation, and applied linear elastic fracture mechanics (LEFM) to determine the fracture toughness of these materials. We will address issues such as loading configuration for stable crack growth, diagnostics for identifying crack initiation and quantifying the extent of crack growth, and scheme for extracting the stress intensity factor at the moving crack tip. The technique described in this report has been applied to many different materials, but for the purpose of illustrating the application of the technique and data processing scheme, we choose the following materials: graphite and beryllium as materials described in this paper. This technique can be used at any rate with the only limitation being the resolution and rate at which images can be captured.
\end{abstract}

\section{Introduction}

The primary reason for this investigation was to use the technique developed to determine the initiation of cracking[1], measure the crack location and growth rate, and quantitatively determine the fracture toughness. In order to do this there needs to be stable crack growth, a way of determining the crack location, and a way to relate the crack properties to a far-field applied stress. From this relationship the stress intensity factor (fracture toughness) can be determined. One necessity of this test technique is that when subjected to overall compressive load, tensile cracks can still form inside the material, a phenomenon which is attributed to the existence of heterogeneity within the material such that local tensile stress can arise around the heterogeneity.

Our specimen geometry is a rectangular block with a circular hole in the center of a block. A compressive load is applied along the long axis of the block. This geometry has been shown to be effective on several other materials [2-7]. This technique was first used on transparent materials $[2,3]$ and later on opaque materials [4-7]. For the opaque materials the

\footnotetext{
* Corresponding author: cady@lanl.gov
} 
determination of the crack tip and the extent of cracking has to me made in order to determine fracture toughness.

Several benefits of this technique is that small test samples can be used for many common materials and it can be used to measure elastic response of the material during early test conditions. The loading fixture is quite simple and only involves uniaxial compression, and can be characterized with most load frames including small systems with limited load capacity. This specimen design creates stable crack growth, and can be used investigate the stress intensity factor at the moving crack tip as a function of the crack length since for a relatively long crack the fracture toughness is a material property and is independent of crack length. Finally, model development and validation of the test can be easily implemented due to the clean and relatively simple boundary conditions.

Section 2 will describe the specimen design, the materials to be tested, the test procedures, the overall response and the displacement field evolution (using DIC). Section 3 describes the method for quantitatively identifying the location and extent of damaged and cracked regions, the correlation coefficient. This value is used to determine the boundary between damaged and undamaged regions in brittle/quasi-brittle materials, thus enabling for the determination of properties of the crack, the tip location, opening and boundary. By doing a frame by frame analysis the crack initiation and growth rate can be determined. The brief discussion about the stress intensity factor and references to more detailed evaluation will be described in the next section. Finally, the discussion of the experimental results and conclusions will end this paper.

\section{Specimen, experimental method, test material, crack evolution}

The "compression - fracture" (CF) sample geometry used in this experimental investigation is a rectangular plate with a circular hole at the center of the plate. The specimen and the loading configuration are shown in Fig. 1(a). The dimension of the specimen is characterized by the height $H$, the width $W$, the thickness $B$, and the diameter of the circular hole $d$. For materials that are more ductile in nature a modification to the sample design may be necessary (Fig.1(b)). With the only difference being that $L_{0}$ is used in place of $d$ for the initial crack length. A compressive load, $P$, is applied to the sample which generates tensile stresses at the top and the bottom edges of the circular hole due to stress concentrations. When the tensile stress is high enough cracks will form at those two locations and the cracks will propagate into the specimen as the overall deformation continues to increase. These cracks are schematically illustrated with $a_{\text {top }}, a_{\text {bottom }}$, and sometimes with $L$ to characterize the total crack length. The applied load $P$ and displacement $\delta$ are recorded by the load frame and synchronized to the recorded images using the load signal.

A conventional servo-hydraulic load frame MTS 880 was used to load the specimens at a constant a nominal strain rate of $\sim 7.0 \times 10^{-5} /$ second. The applied compressive load $P$ and the crosshead displacement $\delta$ were monitored and recorded at the sampling rate of $10 \mathrm{~Hz}$. For applying the DIC technique, a random speckle pattern is applied to the specimen surface by first spraying a thin layer of white paint as background and then spraying black paint for generating random speckles. A CCD camera, with resolution of $2448 \times 2048$ pixels, used for image capture at a rate of 4 frames/second with the load signal synchronized to the frames. This produces a physical resolution of $12 \mu \mathrm{m} /$ pixel. No lubrication is used in the contact surface between the sample and the loading surface.

The materials chosen for this investigation are graphite and beryllium. The graphite was a high density Mersen product with a density of $1.77 \mathrm{~g} / \mathrm{cm}^{3}$ made by isostatic pressing. It has a nominal Young's modulus of $\mathrm{E}=10.7 \mathrm{GPa}$ and a porosity of $\sim 9 \%$ with an average grain size is about $15 \mu \mathrm{m}$ [1]. The dimensions of the graphite specimen shown in Fig. 1(a) has the nominal dimensions, $H=28.6 \mathrm{~mm}, W=9.5 \mathrm{~mm}, B=6.4 \mathrm{~mm}$, and $d=3.0 \mathrm{~mm}$. The beryllium 
was a hot isostatically pressed S-200F beryllium from Brush Wellman, Inc. The total impurity level for this grade is typically below $1 \%$ and while the grain size varies considerably, the average is $\sim 11 \mu \mathrm{m}$ [8]. The nominal dimensions or these tests were $W=$ $9.5 \mathrm{~mm}, H=25.5 \mathrm{~mm}, B=4.8 \mathrm{~mm}, d=3.2 \mathrm{~mm}$, and $L_{0}=3.6 \mathrm{~mm}$.

For expediency only one example will be given for the determination of deformation and damage as it evolves in materials. First the applied load, $P$, normalized by the initial area of the specimen $B W$ is plotted against the displacement, $\delta$, normalized by the initial height $H$ (Fig.1(c), (d)). The displacement experienced by the specimen was determined using the DIC displacement results, limiting the effects of machine compliance in the analysis. The trend depicted in Fig. 1(c) and (d) is shows that there is a linear elastic initial loading followed by a gradually decreasing slope in load versus displacement.

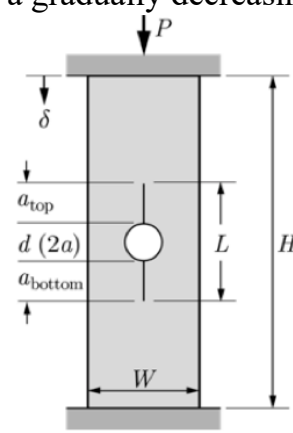

(a)

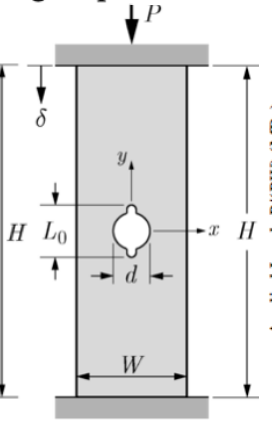

(b)

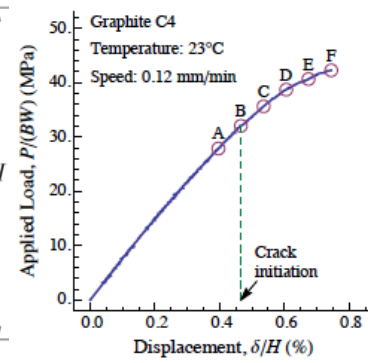

(c)

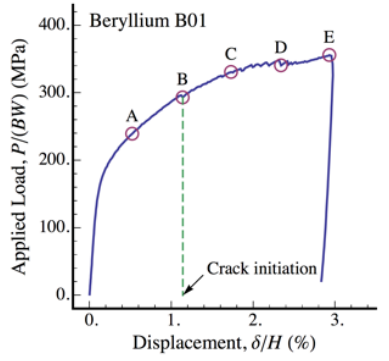

(d)

Fig. 1. Schematic of the compression fracture test geometries for a) graphite, and b)beryllium and examples of load displacement curves for c) graphite and d) beryllium. Indicators that correspond to sequential strain and correlation maps in the next figure. Location B is the moment when the correlation value begins to change rapidly.

The next step in the process is to calculate the deformation fields using standard DIC techniques described elsewhere [1,9]. The displacement field over the sample surface was obtained during the test by comparing each random speckle image against the initial image. The displacement field on the sample surface at those selected moments indicated in Fig. 1(c) A through F, where $u_{x}$ is the displacement in the horizontal direction and $u_{y}$ in the vertical direction. At moments A and B there is very little local displacement seen, but at the moments $\mathrm{C}$ and $\mathrm{D}$ the contour lines become much closer together near the hole as seen in the $u_{x}$ and $u_{y}$ displacement fields (Fig. 2). As the load continues to increase (moments E and F) deformation becomes even more apparent.

The strain fields are computed using numerical differentiation of the displacement fields will sometimes indicate the presence of a crack. In this case, when cracks are evident in the $u_{x}$ displacement field they are often observed in the $\varepsilon_{x}$ field with concentrated strain component, $\varepsilon_{x}$, is above and below the hole suggestive of a formation of a tensile crack. However, the presence of the crack is not as apparent in the other strain fields. Therefore, depending on the orientation of the cracks, some components of the deformation field may clearly indicate the existence of growing cracks, while others may not be sensitive to the presence of cracks. 


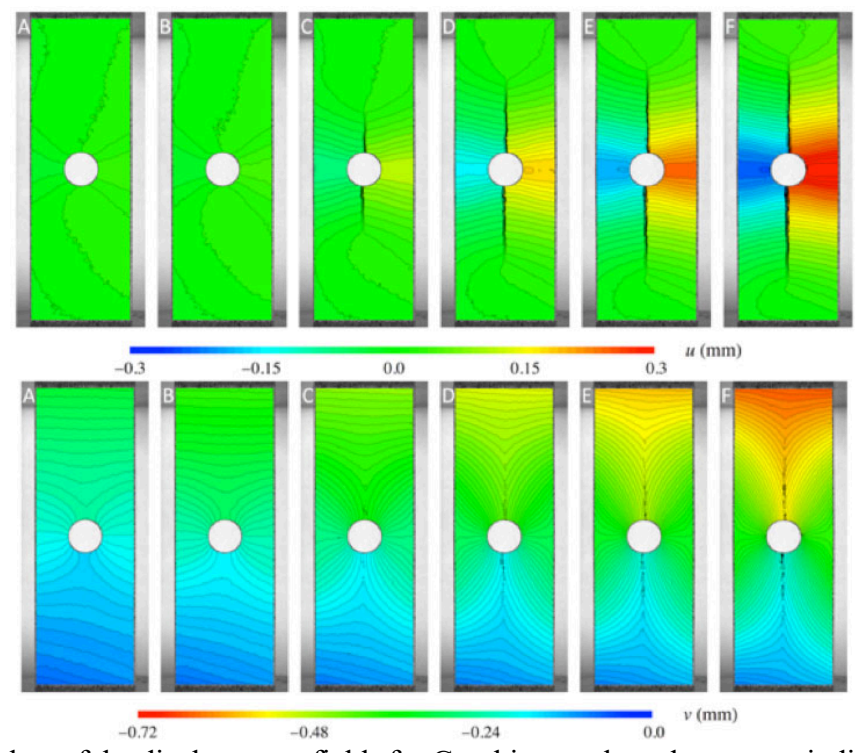

Fig. 2. Contour plots of the displacement fields for Graphite at selected moments indicated in Fig. 1(c). Displacement $u_{x}$ in horizontal direction (top) and displacement $u_{\mathrm{y}}$ in vertical direction (bottom).

\section{Crack identification and damage extent}

In this section, a simplified explanation of technique for identifying the cracked region and crack tip location based on the correlation coefficient, which is provided by the DIC calculation is presented. Using the technique to determine the correlation coefficient it is possible to define the location and onset of damage initiation. Images corresponding to point "B" give an indication of the onset of damage (as seen in the correlation maps) with further crack growth progressing to and beyond position "F" (Fig 3). By analyzing all images using

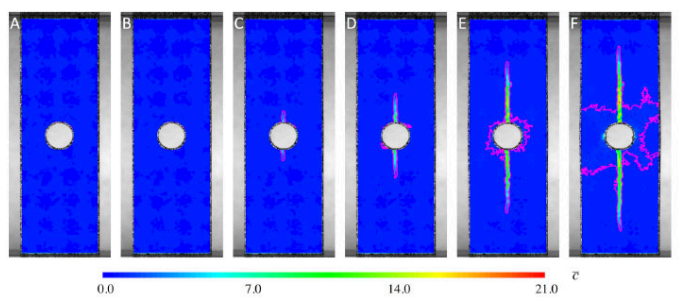

a)

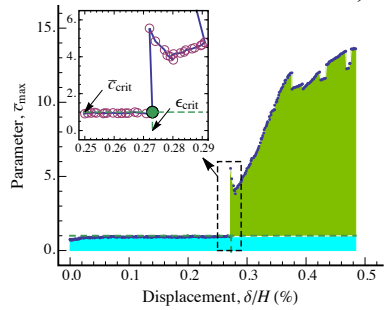

b)

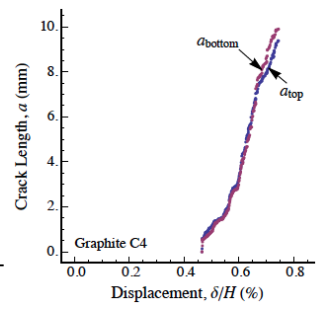

c)

Fig. 3. Contour plots of the correlation coefficients at moments indicated in Fig. 1(a) (left), the development of the correlation coefficient during a test (center - b ), and crack length and a function of displacement (right $-\mathrm{c}$ ). 
this technique it is possible to observe that there are two dominant cracks present, one propagating upward and the other down. This methodology is used to generate a unique correlation for every frame of the video that captured the test, thus, providing information about crack location, length, and growth rate.

It is necessary to elaborate on the technique used to determine the initiation and boundary of the cracks. This is done first by analyzing the maximum correlation value as it evolves during a test (Fig 3(b)). This plot shows a relatively flat, unchanging, value during the early part of the test, typically associated with the elastic loading of the sample. At the time when a crack initiates and begins to grow the correlation value also increases rapidly. The correlation value where this transition occurs is called the critical correlation coefficient. This coefficient will be used to track the boundary of the "damaged" region showing where the crack is located and the rate at which it grows for the entire duration of the test. The maximum correlation value is not specifically related to a single point but once a crack is initiated the correlation value of that point will increase rapidly and this curve can be thought of as an accurate representation of the correlation coefficient at the damage initiation site. Typically, the maximum correlation value is associated with the region, if not exact position, of the onset of damage and the perimeter of the damage region can be traced when the value of correlation exceeds the critical correlation value.

The crack may not grow uniformly in both directions during the test but the analysis for the crack can be done independently. (Fig. 3(c)). The load versus displacement and the crack length versus displacement as analyzed from the correlation map (Fig. 3(a)) shows this non uniform crack growth.

\section{Stress intensity factor for a growing crack}

The applied load, $P$, varies linearly as a function of the deformation, $\delta$, for loads less than a critical value (Fig 4a). This will be definition of critical stress, $\sigma_{\text {crit }}$ and critical deformation, $\varepsilon_{c r i t}$, when crack initiation occurs. The deviation from the linearity starts near the critical moment, which suggests the initiation motion of the growing crack. This indicates that all material elements within the specimen, except those very close to the crack tip, experience elastic deformation. As a result, Linearly Elastic Fracture Mechanics (LEFM) theory can be applied to analyze the experimental data.

The displacement field measurement, obtained using DIC, over the surface of the specimen forms the basis of our data analysis. Consider a particular moment during the compression of a specimen, as shown in Figure 4(b), where two tensile cracks have initiated and extended. For each tensile crack, we choose a coordinate system $\left(X_{1}, X_{2}\right)$ originating at the crack tip, determined according to our experimental scheme, the $X_{1}$-axis is parallel to the loading axis, or in the crack growth direction, and the $X_{2}$-axis is perpendicular to the loading axis. A rectangular region that surrounds each tensile crack is identified with (I and II). The displacement field surrounding the tensile crack at one end of the sample is shown in Figure 4(c), where $U_{1}$ is the component of strain (displacement) along the $X_{1}$-direction and $U_{2}$ is the component of strain (displacement) along the $X_{2}$-direction. We also assign that (wording) $\left(U_{1}\right.$ $\left.=U_{2}=0\right)$ at the origin of the $\left(X_{1}, X_{2}\right)$ coordinate system. 


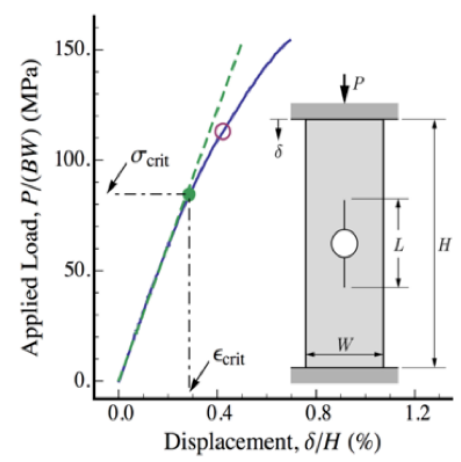

a)

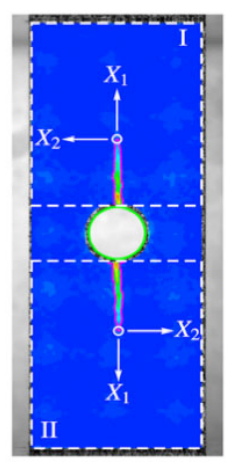

b)
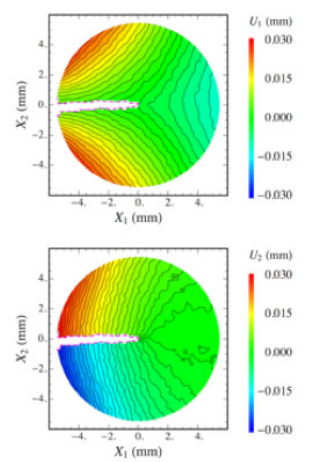

c)

Fig. 4. a) Transition from elastic loading to nonlinear loading at a "critical" moment, b) partitioning of the cracks propagating from the center hole, and c) displacement maps in the "new" coordinate system for one end of the test sample.

A mathematical representation of the displacement field surrounding the crack is needed to extract crack tip parameters. This representation is usually expressed in the crack-tip coordinate system, as shown in Figure 5. The $\left(x_{1}, x_{2}\right)$ system is originated at the crack tip and the $x_{1}$-axis is tangent to the crack at the crack tip and $x_{2}$-axis is perpendicular to the crack.

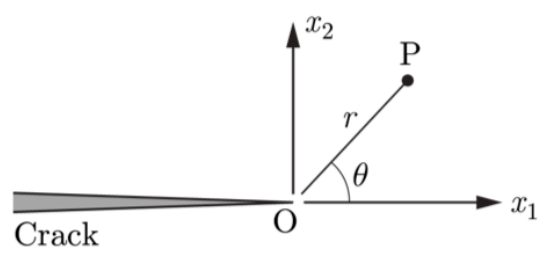

Fig. 5. Crack-tip coordinate system.

According to the asymptotic analysis of the elastic fracture mechanics [10], the displacement field, written in the complex form $u_{1}+i u_{2}$, can be represented in the series form,

$$
\begin{aligned}
u_{1}+i u_{2}=\frac{1}{2 \mu} \sum_{n=0}^{N}\{ & \left\{\frac{2 \kappa A_{n}}{2 n+1} z^{n+1 / 2}-\left[\frac{2 A_{n}}{2 n+1}+\left(\frac{z^{2}}{|z|^{2}}-1\right) \bar{A}_{n}\right] \bar{z}^{n+1 / 2}\right. \\
& \left.+\frac{\kappa B_{n}}{n+1} z^{n+1}-\left[\frac{B_{n}}{n+1}+\left(\frac{z^{2}}{|z|^{2}}+1\right) \bar{B}_{n}\right] \bar{z}^{n+1}\right\}+O\left(|z|^{N+3 / 2}\right),
\end{aligned}
$$

Where $z=x_{1}+i x_{2}, \mu$ is the shear modulus of the material, and parameter $\kappa$ is related to the Poisson's ratio $v$ through

$$
\kappa=\left\{\begin{array}{cl}
(3-v) /(1+v), & \text { for plane stress, } \\
3-4 v, & \text { for plane strain. }
\end{array}\right.
$$

$A_{n}$ and $B_{n}$ are the coefficients of the asymptotic expansion and they cannot be determined by the asymptotic analysis itself but by the far-field conditions. A bar over a symbol stands for the complex conjugate.

Equation (1) gives the complete representation of the displacement field surrounding a crack for $N \rightarrow \infty$. However, very often only the leading term, or the most singular term, is used to illustrate the near-tip deformation field structure, where the displacement field is characterized by a single complex number $A_{0}$ by 


$$
u_{1}+i u_{2}=\frac{\kappa A_{0}}{\mu} z^{1 / 2}-\frac{1}{2 \mu}\left\{2 A_{0}+\left(\frac{z^{2}}{|z|^{2}}-1\right) \bar{A}_{0}\right\} \bar{z}^{1 / 2}
$$

If we express the complex coefficient $A_{0}$ in terms of the stress intensity factors, $K_{\mathrm{I}}$ and $K_{\mathrm{II}}$, through

$$
A_{0}=\frac{K_{\mathrm{I}}-i K_{\mathrm{II}}}{2 \sqrt{2 \pi}}
$$

and by using the polar representation $z=r e^{i \theta}$, where $r=\left(x_{1}^{2}+x_{2}^{2}\right)^{1 / 2}$ and $\theta=$ $\tan ^{-1}\left(x_{2} / x_{1}\right)$, then the near-tip displacement field can be expressed in a more familiar form,

$$
\begin{aligned}
& u_{1}=\frac{K_{\mathrm{I}}}{2 \mu \sqrt{2 \pi}} r^{1 / 2} \cos \frac{\theta}{2}\left(\kappa-1+2 \sin ^{2} \frac{\theta}{2}\right) \\
&+\frac{K_{\mathrm{II}}}{2 \mu \sqrt{2 \pi}} r^{1 / 2} \sin \frac{\theta}{2}\left(\kappa+1+2 \cos ^{2} \frac{\theta}{2}\right), \\
& u_{2}=\frac{K_{\mathrm{I}}}{2 \mu \sqrt{2 \pi}} r^{1 / 2} \sin \frac{\theta}{2}\left(\kappa+1-2 \cos ^{2} \frac{\theta}{2}\right) \\
&-\frac{K_{\mathrm{II}}}{2 \mu \sqrt{2 \pi}} r^{1 / 2} \cos \frac{\theta}{2}\left(\kappa-1-2 \sin ^{2} \frac{\theta}{2}\right) .
\end{aligned}
$$

Finally, the energy release rate at the crack tip, $\mathcal{G}$, is related to the stress intensity factors through

$$
\mathcal{G}=\frac{\kappa+1}{8 \mu}\left(K_{\mathrm{I}}^{2}+K_{\mathrm{II}}^{2}\right), \quad \text { or } \quad \mathcal{G}=\frac{\pi(\kappa+1)}{\mu} A_{0} \bar{A}_{0} .
$$

For a growing crack, the energy release rate $\mathcal{G}$, determined according to Eq. (4), represents the fracture toughness of the testing material, i.e., $\mathcal{G}=\mathcal{G}_{\mathrm{C}}$. For mode-I cracks, the critical stress intensity factor $K_{\mathrm{IC}}$ sometime is also called fracture toughness. The goal of this section is to extract the stress intensity factors, $K_{\mathrm{I}}$ and $K_{\mathrm{II}}$, based on the measurement of displacement field surrounding the crack tip.

A least square fitting routine is used to solve for the energy release rate, or fracture toughness. This process is detailed elsewhere [1]. In summary, this process involves fitting the displacement field, $u_{1}$ and $u_{2}$ obtained from DIC measurement as shown in Figure 4c, to Eq. (1) for a fixed number of terms $N$ to obtain the coefficients $A_{n}$ and $B_{n}$, where $n=$ $0,1, \cdots, N$. Then the number of terms $N$ gradually increases until the leading term coefficient $A_{0}$ converges. Note that the stress intensity factors at the crack tip, $K_{\mathrm{I}}$ and $K_{\mathrm{II}}$, are related to the coefficient $A_{0}$ according to Eq. (4), therefore, the stress intensity factors at the crack tip are determined. For a growing mode-I crack, the measured stress intensity factor $K_{\mathrm{I}}$ at the crack tip also represents the fracture toughness $K_{\mathrm{IC}}$, a characteristic parameter that describes the ability of a material to resist fracture.

\section{Experimental evaluation}

Typical load displacement responses for the two materials in this study are shown Figure 6. From these plots it can be concluded that the test results are reproducible with acceptable differences in their loading behavior for all loading rates. Several repeat tests for each material indicate that the onset of damage occurs at nominally the same load level. 


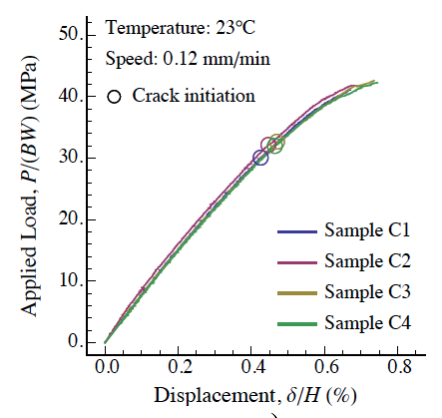

a)

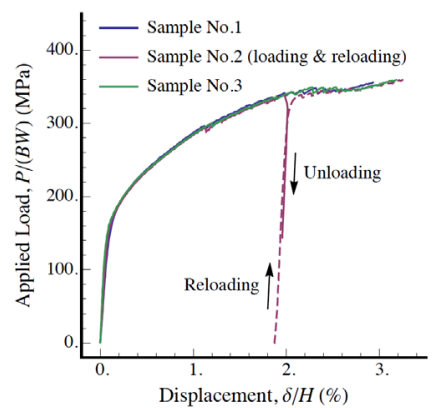

b)

Fig. 6. Typical load displacement curves for a) graphite, and b) beryllium,

Typical plots for the LEFM solution for these tests are presented below (Fig. 7). These plots provide insight into the development of fracture toughness. If the crack is arrested the value of $\mathrm{K}_{1 \mathrm{C}}$ increases, when it is growing quickly, $K_{1 \mathrm{C}}$ drops. Uniform crack growth reduces the magnitude of the scatter and there is a minimum crack length for uniform crack growth on the order of the initial hole radius. For graphite, the fracture toughness $K_{\text {IC }}$ becomes approximately constant as the crack grows longer than about one radius. From these four tests we see a fracture toughness from $\sim 1.1 \pm 0.15 \mathrm{MPa} \sqrt{\mathrm{m}}$. In the tougher beryllium material there is more "stick-slip" in the failure process leading to a wider distribution in $K_{\text {IC. }}$ First the value of fracture toughness is much higher. There is more variability from sample to sample and the spread in the value is higher. For three samples tested we had average $K_{1 \mathrm{C}}$ values of $71.5,109$, and $124 \mathrm{MPa} \sqrt{ } \mathrm{m}$ with a spread around $\pm 22 \mathrm{MPa} \sqrt{\mathrm{m}}$.

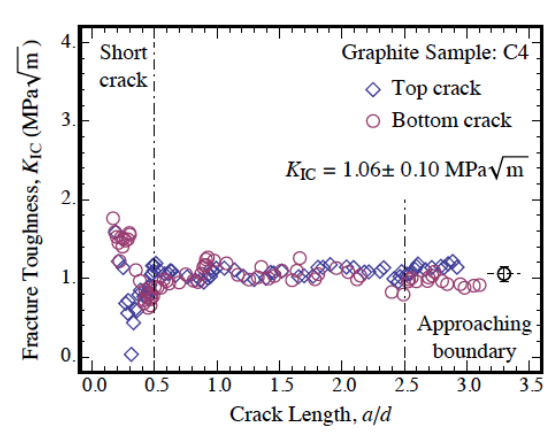

a)

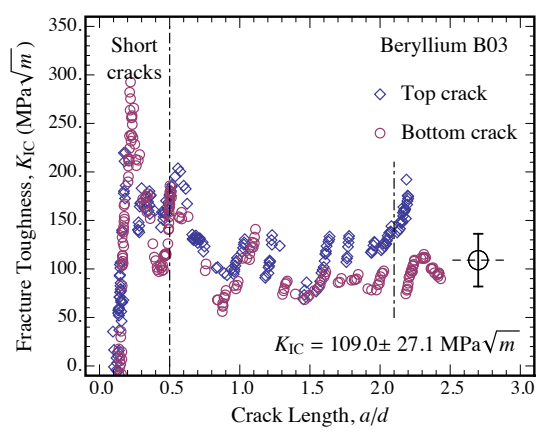

b)

Fig. 7. Example of LEFM solution for fracture toughness $\left(\mathrm{K}_{1 \mathrm{C}}\right)$ in a) graphite and b) beryllium.

\section{Conclusions}

The compression-fracture test geometry is suitable for observing and measuring the cracking process, including initiation and propagation, in brittle and quasi-brittle materials. An advantage of this sample geometry is that the mode-II stress intensity factor $K_{\text {II }}$ is essentially zero due to the deformation state near the crack tip is predominantly mode-I, though some deviation from zero is observed because of the heterogeneous nature of the material and crack path is not exactly a straight line. The method for extracting the fracture toughness, $K_{1 \mathrm{C}}$, at the crack tip is presented with details described in other references $[1,6]$. This technique uses coordinated testing and evaluation techniques involving high speed imaging, digital image correlation, and standard data acquisition from the load frames with the only limitation of the analysis being subject to the capabilities of the equipment available. 
The test configuration involves the compression of a rectangular block with a circular hole at the center of the block. One advantage of using this specimen design is that specimen sizes can be reasonably small. Using DIC and the correlation coefficient, it is possible to identify the crack boundary, the crack tip, and a crack tip velocity. The quantitative relationship between the applied load and the characteristics of the growing cracks can be established and by using the displacement field information of DIC and LEFM analysis a stress intensity factor can be computed using a least-squares fitting routine. This fracture toughness $K_{1 \mathrm{C}}$ determined from the tests is a reasonably constant for a relatively long cracks in graphite with more variability seen in the beryllium. Uncertainties in the value for fracture toughness calculated using LEFM come from two sources. First, the scatter found within the analysis of a single test, and second, sample to sample variation. It is thought that a representative sample size is necessary to randomize the material being tested that will produce more uniform test results. Clearly, there is sufficient material available for the graphite material. However, it is apparent that larger sample sizes ought to be tested for beryllium to see if the variability in the value for $K_{1 \mathrm{C}}$ and the amplitude of the uncertainty could be reduced.

One of the benefits of using image capture and DIC is that it allows for re-evaluation of the test at any time in the future since the entire test is recorded for evaluation. The both materials exhibited easily traceable cracks and easily measurable crack growth rates that allowed for consistent calculation of the fracture toughness using the analysis technique.

Acknowledgements: Los Alamos National Laboratory is operated by Triad National Security, LLC, for the National Nuclear Security Administration (NNSA) of U.S. Department of Energy (Contract No. 89233218CNA000001). Programs that have supported this work include LDRD, Life Extension, Enhanced Surveillance, Science and Engineering (C2), and Joint Munitions.

\section{References}

1. C. Liu, C.M. Cady, "Observations of Cracking and Measurements of Fracture Toughness in Brittle/Quasi-Brittle Solids," Los Alamos National Laboratory Report, LA-UR-20-28805, 2020.

2. C.G. Sammis and M.F. Ashby, Acta Metall., Vol 34(3) pp511-526 (1986)

3. T.A. Plaisted, A.V.Amirkhizi, S. Nemat-Nasser, I J Fract, 141 pp447-457 (2006)

4. C.M. Cady, C. Liu and M.L. Lovato, EPJ, 94, 01012 (2015).

5. C. M. Cady, D. E. Eastwood, N. K. Bourne, C. Liu, R. Pei, P. Mummery, W. Bodel, J. Wade, R. Krishna, S. Cipiccla, A. J. Bodey, K. Wanelik, and C. Rau, AIP conference proceedings, 2018-07-03, Vol.1979 070009 (2018).

6. C. Liu, C.M. Cady, and K.J. Ramos, 16th International Detonation Symposium, pp226236 (2018).

7. C. Liu, C.M. Cady, and K.J. Ramos, 16th International Detonation Symposium, pp1614-1623 (2018)

8. W.R. Blumenthal, R.W. Carpenter, G.T. Gray III, D.D. Cannon, S.P. Abeln, AIP Conf. Proc. 429 pp411-414 (1998)

9. M.A. Sutton, S.R. McNeill, J.D. Helm, Y.L. Chao, Topics Appl. Phys. 77 pp323-392 (2000)

10. J.R. Rice, Fracture - An Advanced Treatise, Vol. II, H. Liebowitz (ed.), Academic, New York, pp.191-308, 1968. 